\title{
Nematic Order in Polyesters with Flexible Moieties in the Main Chain
}

\author{
Alexandre BLUMSTEIN \\ Polymer Science Program, Chemistry Department, \\ University of Lowell, Lowell, MA 01854, U.S.A.
}

(Received August 20, 1984)

\begin{abstract}
Properties of polyesters based on 4,4'-dihydroxy-2,2'-dimethylazoxybenzene and a homologous series of alkanedioic acids with methylene sequence length $n=3-4$ are discussed in the light of recent results based on DSC, PMR, magnetic birefringence and X-ray data. Influence of substitution in the mesogen and spacer is also described. Particular attention is given to the oddeven effect in the homologous series and the existence of two distinct levels of molecular order for the $n=$ even and $n=$ odd members of the series. On the basis of X-ray diffraction data performed on oriented samples, a cybotactic micellar model for the $n=$ even polymers is proposed. The cooperative nature of the formation of cybotactic micelles is shown by the study of order parameter and its dependence on the degree of polymerization. Progressive development of layered order in $n=$ even polymers with increase in molecular mass is also shown by SAX patterns of unoriented samples of poly(4,4'-dioxy-2,2'-dimethylazoxybenzenedodecanedioyl). While the Meier and Saupe theory of the nematic state is in good agreement with the $S(T)$ dependence for noncybotactic polymer systems for most of the nematic interval, it diverges in the vicinity of the clearing point. In contrast to low molecular mass nematics, polymeric nematics show a remarkable stability at the clearing temperature and a large pretransitional interval.

KEY WORDS Nematic Order / Flexible Thermotropic Polyesters / Cybotactic Nematic Polyesters / Odd-Even Effect at the N/I Transition / $\mathrm{N}+$ I Bi-Phase /
\end{abstract}

In this paper, properties of a family of thermotropic nematic main chain polymers with regularly alternating rigid mesogenic and flexible moieties are described. An impressive volume of work has been obtained for such systems over the past few years and was reviewed. ${ }^{1-3}$ However, inadequate knowledge of sequence distribution, homogeneity and molecular weight distribution of polymer samples often lead to mistaken conclusions about liquid crystalline behavior and structure correlations. A need for a well characterized system is therefore of prime importance. We believe that the polymers described in this paper, derived from condensation of 4,4'-dihydroxy-2,2'-dimethylazoxybenzene (mesogen 9) and alkanedioic acids provide such a system:

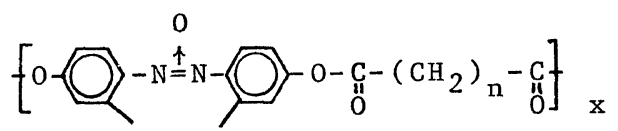

The moderate transition temperatures of the dimethylazoxybenzene moiety (minimizing decomposition and/or ester interchange reactions) combine with a strong mesogenic tendency of the azoxy group to provide a family of linear polyesters that is particularly well suited for study and understanding of flexible main chain polymeric liquid crystals.

The aim of this article is to discuss the evolution of properties of these compounds in the light of our recent results. Attention will be given to the structural parameters affecting the formation, stability and the degree of order of the nematic mesophase. The conformation of nematic polyesters in the isotropic, nematic and oriented nematic state will be discussed and evidence for two distinct levels of order in polymeric nematic mesophases will be presented. 


\section{EXPERIMENTAL}

The experimental techniques used to obtain data discussed in this paper are given in the original references cited herein. The synthesis of polyesters based on 4,4'-dioxy-2,2'-dimethylazoxybenzene and alkanedioyl spacers by interfacial and solution polycondensation is given in ref 7-9. The synthesis of oligomers and models is given in ref 23. Fractionation of AZA-9 was done by fractional precipitation using DMF-ethanol solventprecipitant couple. DDA-9 was fractionated from DMF solution using a temperature gradient. The molecular weights were measured using a combination of vapor pressure osmometry, viscometry, DSC and end group analysis by NMR as described in ref 4,7 , and 11 .

Differential Scanning Calorimetry was used to determine the temperatures, enthalpies and entropies of phase transition using a Perkin Elmer DSC-2C calorimeter. The measurements were performed as described in ref 4,7 , and 9 .

$\mathrm{X}$-ray diffraction (XRD) was performed using a Laue camera as in ref 11 and 12. SAX patterns were recorded using a Rigaku-Denki scanning diffractometer as described in ref 7 . The nematic order parameter was measured by PMR as described in ref 14. Techniques for magnetic birefringence measurements and quenching of aligned samples were described in ref $7,11,15$, and 16. Electrohydrodynamic instabilities and miscibility studies were described in ref 19 and 21 .

\section{RESULTS AND DISCUSSION}

\section{Nematic-Isotropic Biphase: Influence of Molecular Mass and End Groups}

In contrast to low molecular mass liquid crystals (LMLC), usually composed of single molecular species, polymers and oligomers displaying liquid crystalline properties (PLC) are characterized by a variety of molecular species due to polydispersity of molecular mass, compositions (copolymers), chain ends and isomers. The result is the appearance of a nematic-isotropic $(\mathrm{N}+\mathrm{I})$ biphase the width of which depends on the average molecular mass and global polydispersity of the sample. The sharp clearing points of LMLC should therefore be replaced in PLC by average values. Figure 1 gives the appreciation of the $\mathrm{I} / \mathrm{N}$ phase transition of a sample

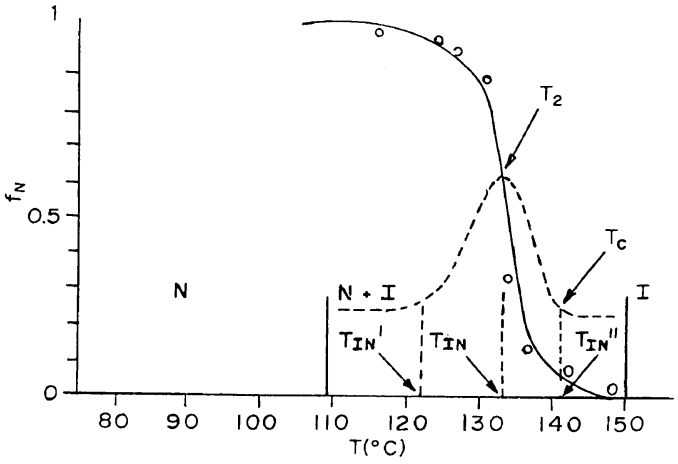

Figure 1. Width of a biphase as given by PMR, DSC and polarizing microscopy. Sample DDA-9, unfractionated $M_{n}=4000$. PMR gives the weight fraction of the nematic phase $f_{\mathrm{N}}$ (solid curve). DSC gives the exotherm associated with the $\mathrm{I} \rightarrow \mathrm{N}$ transition (dashed curve). $T_{\mathrm{c}}$, temperature at which the last nematic droplets disappear on heating; $T_{\mathrm{IN} \text { ", }}$ temperature at which the first nematic droplets appear on cooling; $T_{\mathrm{IN}^{\prime}}$, temperature at which the last isotropic droplets disappear on cooling; $T_{2}$, temperature at which the first isotropic droplets appear on heating. Scanning rate $10^{\circ} \mathrm{C} \mathrm{min}-1$, thermal history as in reference 4 .

of poly(4,4'-dioxy-2,2'-dimethylazoxybenzenedodecanedioyl) (DDA-9) of $M_{n}=4000$ (on cooling) by three methods: 1) by polarizing microscopy: the biphase is characterized by $T_{\mathrm{c}}-T_{2}$, where $T_{\mathrm{c}}$ is the temperature of the first birefringent droplet; $T_{2}$ corresponds to the disappearance of the last isotropic droplet; 2) DSC: the biphase is characterized by the width of the exothermic peak (on cooling) $T_{\mathrm{IN}^{\prime \prime}}-T_{\mathrm{IN}^{\prime}}$; and 3) PMR: the biphase is characterized by the nematic fraction $f_{\mathrm{N}}$ obtained on slow cooling from the isotropic phase. ${ }^{4}$

One can easily see from Figure 1 that the range of the biphase delineated by the NMR experiment (interval between the two vertical lines) is larger than the range delineated by $\operatorname{DSC}\left(T_{\mathrm{IN}^{\prime \prime}}-T_{\mathrm{IN}^{\prime}}\right)$ or polarizing microscopy $\left(T_{\mathrm{c}}-T_{2}\right)$. One can note, however, that the maximum on the DSC curve corresponds to the temperature of disappearance of isotropic droplets $T_{2}$ as well as to the inflection point on the $f_{\mathrm{N}}(T)$ curve from PMR and is therefore adopted as the temperature of isotropic-nematic transition $T_{\mathrm{IN}}$. One also notes that PMR provides the most accurate method for measuring biphase intervals as well as the extent of nematic phase (referred sometimes as "degree of liquid crystallinity"). The width of the biphase can as a first 
approximation be expressed at $T_{\mathrm{IN}}-T_{\mathrm{IN}}$ by DSC, which is close to twice $\left(T_{\mathrm{c}}-T_{2}\right)$ by microscopy.

Figure 2 illustrates the variation of biphase width as observed by polarizing microscopy (rate of scanning $10^{\circ} \mathrm{min}^{-1}$ ) for a series of fractions of DDA-9, of relatively narrow molecular mass distribution $\left(M_{w} / M_{n} \sim 1.1-1.2\right)$. The trend is unmistakable: the biphase narrows for high molecular weights. For polymers, comparison of properties should be undertaken for fractions of high molecular mass, exceeding if possible $\overline{\mathrm{DP}}_{n} \sim 20$. In what follows, we have tried, unless stated otherwise, to compare polymers with relatively narrow distribution $\left(M_{w} /\right.$

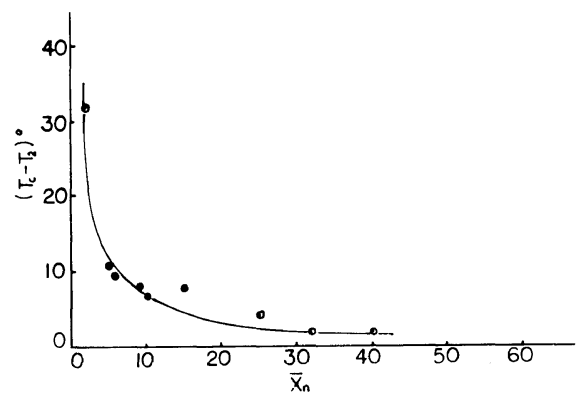

Figure 2. Width of a biphase for DDA-9 polyester observed by polarizing microscopy on heating as a function of the number average degree of polymerization $\bar{X}_{n}$.
$\left.M_{n}<1.3\right)$ and $\overline{\mathrm{DP}}_{n}>15-20$ in order to minimize the influence of the extent and breadth of the biphase.

The rather large biphase observed for $\overline{\mathrm{DP}}_{n}<10$ is due above all to the influence of molecular mass and to a smaller extent to the nature of terminal groups which under conditions of stoechiometric condensation can be terminated by mesogen 9 (aromatic), spacer (aliphatic), or a combination of both. In addition, a mixture of structural isomers such as:
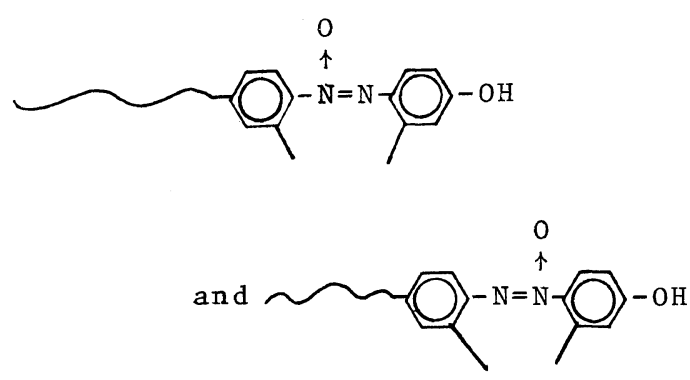

is adding to the magnitude of this influence. ${ }^{4}$ Comparison of properties of liquid crystalline oligomers is delicate and should always consider the nature of end groups.

This influence is apparent in the dependence of $T_{\mathrm{NI}}, \Delta H_{\mathrm{IN}}$ and $\Delta S_{\mathrm{IN}}$ on the molecular mass. In Figure $3, \Delta S_{\text {IN }}$ is plotted against $M_{n}$ for DDA-9 for

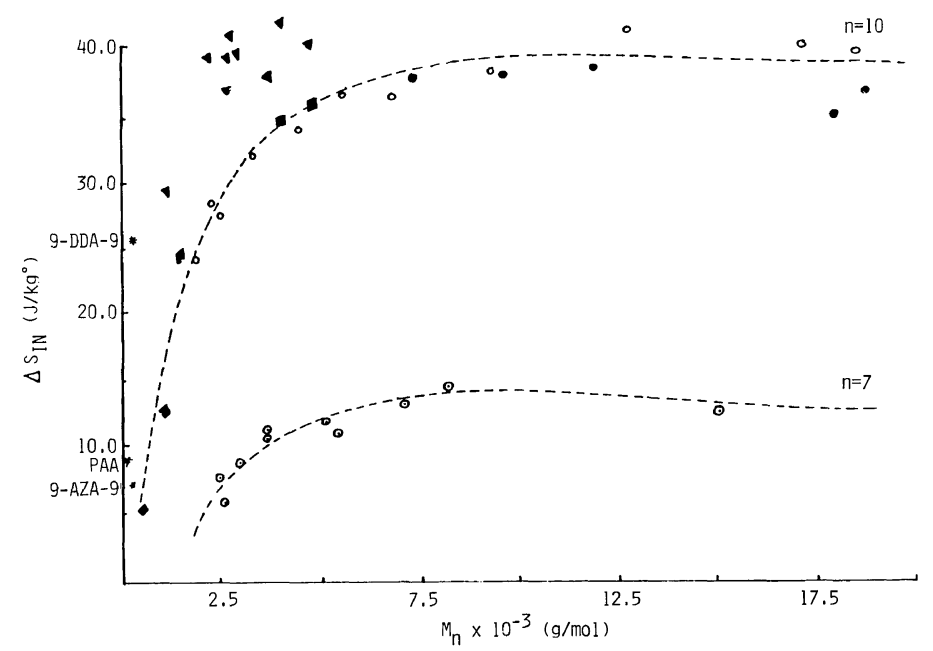

Figure 3. Entropy of isotropization $\Delta S_{\mathrm{IN}}$ as a function of the number average molecular mass $M_{n}$ for DDA-9 $(n=10)$ and AZA-9 $(n=7)$. DDA-9: $\boldsymbol{\Delta}$, samples with predominantly aromatic end groups; $\boldsymbol{\square}$, samples with predominantly aliphatic end groups; $\bullet$, samples with equal numbers of aliphatic and aromatic end groups; $\odot$, unfractionated samples; $\bigcirc$, fractionated samples. AZA-9: $\odot$, fractionated samples. 


\section{A. Blumstein}

various proportions of aromatic/aliphatic end groups $(R)$. The polymer fractions studied were obtained either by precipitations or in the case of low molecular mass and oligomers, by column chromatography with separation occuring largely by end groups type due to preferential adsorption of aromatic (azoxy) end groups. ${ }^{4}$

It can be seen that $\Delta S_{\text {IN }}$ is higher for aromatically terminated oligomers $(R>1)$ than for oligomers with predominantly aliphatic end groups $(R<1)$. The entropy of isotropization increases rather dramatically for low molecular mass and levels off at $\overline{\mathrm{DP}}_{n} \sim 10$. At this value of $\overline{\mathrm{DP}}_{n}$ also, the influence of end groups becomes negligible. On the same graph, the values of $\Delta S_{\mathrm{IN}}$ for fractions of polyester of azalaic acid $(n=7)$ and diol-9 (AZA-9) are plotted. It can be seen that the increase of $\Delta S_{\text {IN }}$ with $M_{n}$ is much smaller and that the leveling occurs here also in the vicinity of $\overline{\mathrm{DP}}_{n} \sim 10$, but at much lower values of $\Delta S_{\text {IN }}$. It should be noted that a "twin" model compound containing a dodecanedioic acid spacer contained between two 4-methoxy-4'-oxy2,2'-dimethylazoxybenzene mesogens (9-DDA-9) gives a $\Delta S_{\mathrm{IN}}$ value intermediate between the fully developed order of DDA-9 polymer and the AZA9 polyester. The "twin" model of 9-AZA-9 gives an even lower value of $\Delta S_{\mathrm{IN}}$ than PAA. The values of $\Delta H_{\mathrm{IN}}$ and $\Delta S_{\mathrm{IN}}$ for AZA-9 are typical of low molecular mass nematic analogs such as PAA $\left(\Delta S_{\mathrm{IN}} \sim 2-4 \mathrm{~J} \mathrm{~mol}^{-1} \mathrm{~K}^{-1}\right)$, while the values for high molecular mass DAA-9 reach isotropization enthalpies and entropies typical of smectic $\mathrm{A}$ or $\mathrm{C}$ systems such as 4,4'-dialkanoyloxyazoxybenzene for $n>7\left(\Delta S_{\mathrm{IN}} \sim 12-18 \mathrm{~J} \mathrm{~mol}^{-1} \mathrm{~K}^{-1}\right)$.

Figure 3 illustrates well the contrast between nematogenic high and low molecular weight liquid crystals and shows the cooperative segmental process leading to the formation of the higher degree of order of the PLC taking place for DP $>8-10$. This order for DDA-9, when fully developed, is characterized by isotropization parameters of low molecular smectic A or C, while AZA-9 displays typically nematic isotropization enthalpies and entropies.

The logical consequence of the rapid variation of thermodynamic mesophase parameters (such as $T_{\text {IN }}$, $\Delta H_{\mathrm{IN}}$, and $\left.\Delta S_{\mathrm{IN}}\right)$ with $M$ is a phenomenon of partitioning by molecular mass between isotropic and nematic phases. Molecules with higher $M$ (higher $T_{\mathrm{IN}}$ and $\Delta S_{\mathrm{IN}}$ ) are expected to be incor- porated into the nematic phase on cooling before molecules with lower values of $M$. This phenomenon may well explain the undulation of the order parameter $S(T)$ observed by Volino et al. ${ }^{5}$ in the vicinity of $T_{\mathrm{IN}}$ on cooling for an unfractionated sample of DDA-9 and absence of such undulation in a fraction of similar molecular weight. Since the nematic order parameter increases with the degree of polymerization $\bar{X}_{n}$ (see Figure 9), the nematic phase in the unfractionated sample incorporates on cooling around $T_{\mathrm{IN}}$ : (see Figure 1) a large fraction of shorter species, thereby producing a large drop in $S$ which is not compensated by the natural increase of $S(T)$ on cooling. The result is an undulation of $S$. This interesting phenomenon indicates a potential possibility of fractionation of flexible main chain thermotropic polyesters of low molecular mass at $\mathrm{N} / \mathrm{I}$ transition.

The segregation of molecular masses in the $\mathrm{N}+\mathrm{I}$ biphase raises also the possibility of a nematic phase composed of domains segregated by chain length ${ }^{5}$ although this point is rather speculative at present.

\section{Structural Considerations}

Influence of Substitution. The liquid crystalline behavior of flexible main chain polyesters of the type described here cannot be explained in terms of theories devised for rigid or semi-rigid molecules. This can be easily seen from Table I, where the transition temperatures, transition intervals for typical samples of thermotropic, main chain polyesters with regularly alternating flexible spacers are given. Remarkably stable and wide mesophases are generated by mesogens with axial ratios far below the critical value of $x \sim 6.42$ for which a phase separation based solely on repulsive entropic interaction is predicted. ${ }^{6}$ Orientation dependent attractive forces operating between mesogenic moieties have to be introduced as an important factor responsible for mesophase formation in these systems. It is through the strengthening or depression of such forces that polymeric mesophases, in analogy to low molecular mass thermotropic mesophases, are stabilized or collapsed. Stability of a mesophase is usually defined as the temperature at which the mesophase appears: the higher this temperature, the more stable the mesophase. This can be seen in Table I providing a sampling of mesophase transition temperatures and intervals depending on substitution for similar mesogens 
Table I. Effect of substitution in the mesogen on mesophase stability and mesophase interval

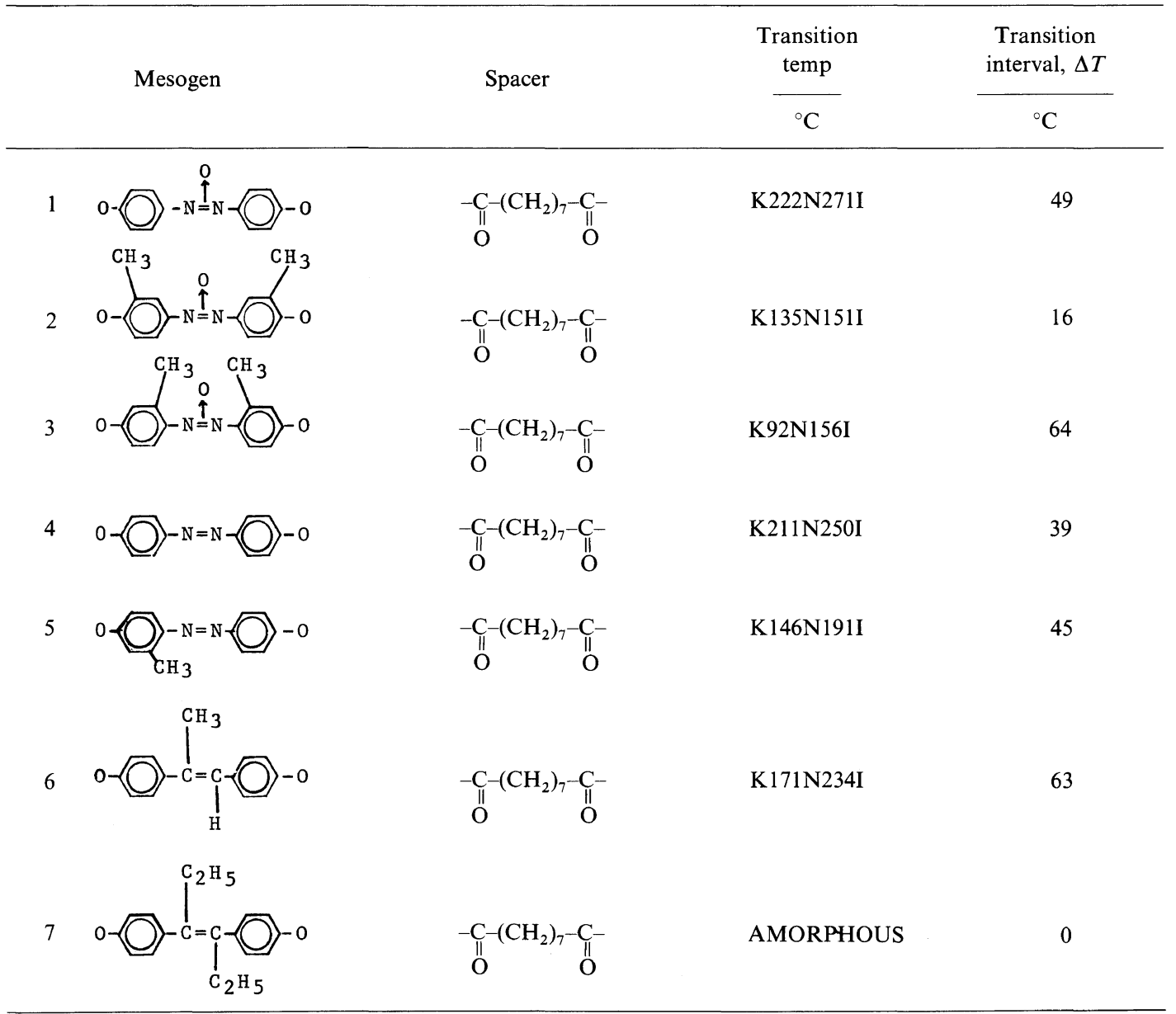

and spacer length $n=7 .^{7}$ Thus, substituted azoxybenzene moieties display in the polymer a far lower mesophase stability than the unsubstituted one. ${ }^{8}$ The same pattern can be seen in the unsubstituted and substituted azobenzene derivatives. It is apparent in Figure 3 that the flexible spacer plays an important role in the formation, nature and stability of the mesophase. This is shown in Table II, which provides a sampling of polyesters based on the same mesogen 4,4'-dihydroxy-2,2'dimethylazoxybenzene (mesogen 9) and mono- and dimethyl substituted alkanedioic acid spacers. It is clear that substitution in the spacer decreases the stability of a nematic mesophase. This simple qualitative result suggests that the alignment of the flexible spacer is a factor in the formation and stability of thermotropic nematic polymeric liquid crystals, and has an important influence on the alignment of the mesogen.

Conformation in the Nematic Phase. The regular and persistent oscillations of the transition temperature $T_{\mathrm{IN}}$, isotropization entropies and enthalpies $\Delta S_{\mathrm{IN}}$ and $\Delta H_{\mathrm{IN}}{ }^{2}$ indicate that conformation of spacer and mesogen are interdependent in the nematic mesophase. Figure 4 shows the oscillations of $\Delta S_{\mathrm{IN}}(n)$ and, superimposed, the oscillations of the order parameter of the mesogen $S(n)$, as determined by PMR in the vicinity of the clearing temperature. It is apparent from Figure 4 that $\Delta S_{\mathrm{IN}}$ and $S$ at $T /$ $T_{\text {IN }} \sim 0.98$ oscillate in unison and that $\Delta S_{\text {IN }}$ is a good 


\section{A. BLumstein}

Table II. Effect of branching in the spacer on mesophase stability

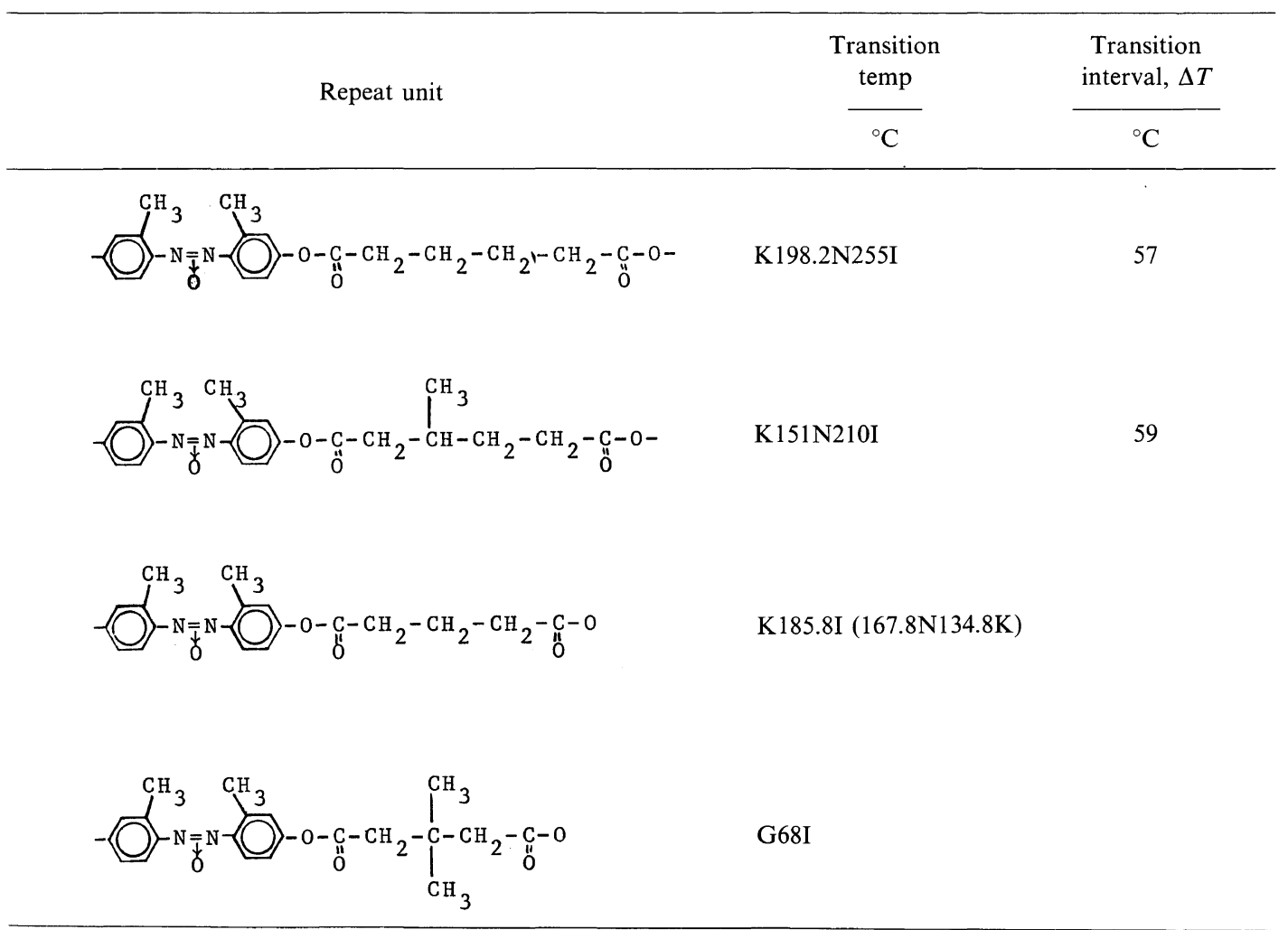

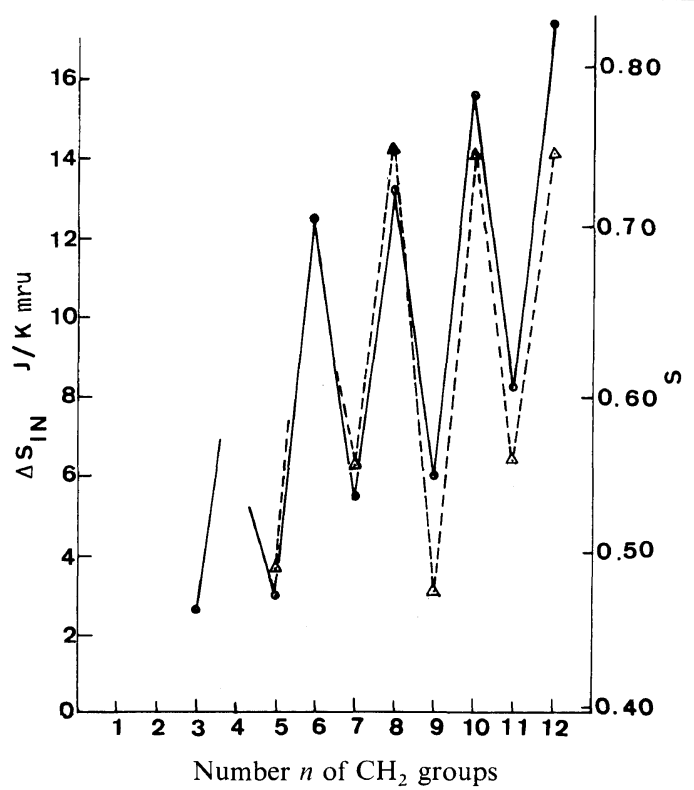

Figure 4. Oscillations of $\Delta S_{\mathrm{IN}}$ (solid line) and of the nematic order parameter $S$ of the mesogen at $T_{\text {red }}=0.98$ (dashed line) with the number $n$ of $\mathrm{CH}_{2}$ groups in the spacer. $\odot, \Delta S_{\mathrm{IN}} ; \triangle, S$. qualitative measure of the degree of mesogen alignment at the phase transition. The extrapolation of lines $\Delta S_{\mathrm{IN}}(n)$ representing the odd and even series ${ }^{9}$ to $n=0$ leads to $\left(\Delta S_{\text {IN }}^{\circ}\right)_{\text {even }}=7.55 \mathrm{~J} \mathrm{~K}^{-1} \mathrm{mru}^{-1}$ and to $\left(\Delta S_{\mathrm{IN}}^{\circ}\right)_{\text {odd }}=1.41 \mathrm{~J} \mathrm{~K}^{-1} \mathrm{mru}^{-1}$.

The intercepts $\Delta S_{\text {IN }}^{\circ}$ represent the entropic contribution of mesogenic groups at the isotropic $\rightarrow$ nematic transition. Consequently, the mesogen is thought of as the 2,2'-dimethylazobenzene moiety augmented by two $-\mathrm{O}-\stackrel{\text { II }}{\mathrm{C}}-$ residues while the flexible spacer is represented by the $-\left(\mathrm{CH}_{2}\right)_{n}$ - sequence. The entropic contribution per methylene unit of the spacer can be obtained from the slopes of the corresponding $\Delta S_{\mathrm{IN}}(n)$ lines and is respectively $\left[\Delta\left(\Delta S_{\mathrm{IN}}\right) / \Delta n\right]_{\text {even }}=0.87 \quad \mathrm{~J}^{-1}$ 和 $\left[\Delta\left(\Delta S_{\mathrm{IN}}\right) / \Delta n\right]_{\text {odd }}=0.57 \mathrm{~J} \mathrm{~K}^{-1}$. If we assume that the entropic contribution of the mesogen $\left(\Delta S_{\mathrm{IN}}^{\circ}\right)$ is orientational in nature, while the entropic contribution per methylene unit of the spacer is conformational, these results suggest that: 1) the degree of alignment of the mesogen in the "even" series is

Polymer J., Vol. 17, No. 1, 1985 
substantially higher than in the "odd" series at the $\mathrm{I} / \mathrm{N}$ transition, approaching values typical of smectic $\mathrm{A}$ or $\mathrm{C}$ rather than of nematic phases; 2) the differences in the degree of alignment of flexible spacers for "odd" and "even" series, although much smaller than those of mesogen moieties, are still noticeably higher in the "even" series. One can easily see from molecular models that in order to align the rigid mesogenic moieties with the nematic director, more conformational trans $\rightarrow$ gauche inversions have to be introduced into the $\mathrm{C}-\mathrm{C}$ bonds of the flexible spacer of the "odd" series as compared to the "even" series. This can be schematically inferred from Figure 5. Thus, small conformational changes in the spacer can lead to substantial differences in the degree of alignment of the mesogen. It is rather interesting to note that $T_{\mathrm{g}}$ determined by DSC (for samples of $M_{n}>10,000$ ) oscillates with $n$ in opposition to the thermodynamic parameters, with the even members displaying lower values than the odd. ${ }^{7}$ One can also note that in contrast to LMLC, the values of supercooling $\Delta\left(T_{\mathrm{IN}}\right)$ of PLC are significant and are, on the average, higher for the $n$ even members of the series than for the $n$ odd ones. These odd-even effects are at present difficult to explain. They are indicative of differences in chain and end group packing of the odd and even members in the nematic mesophase. They point also to morphological differences of nematic polymeric mesophases for both series.

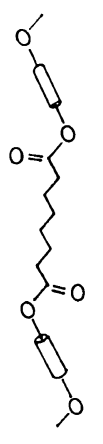

a)

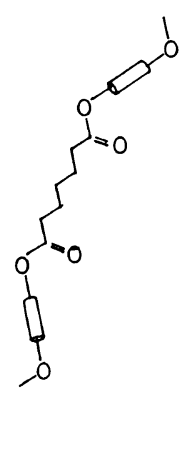

b)
Figure 5. Schematic representation of the conformational spacer-mesogen coupling in the odd-even series: a) Near co-directionality of the mesogens for an even spacer $(n=6)$ in all-trans conformation; b) Disruption of co-directionality of the mesogens for an odd spacer $(n=5)$ in all-trans conformation.
Orientation in Strong Magnetic Fields. Nematic liquid crystals tend to align with an external magnetic field. This alignment is assisted by the anisotropy of magnetic susceptibility of the mesogen, the strength of the field and opposed by thermal motion and the viscosity of the medium. Alignment in fields of 10-15 Tesla could be obtained for $M_{n}<9,000 .{ }^{11,12}$ The low angle pattern of the even series for $n=6,8,10$, and 12 is similar to the oriented cybotactic nematic pattern described by

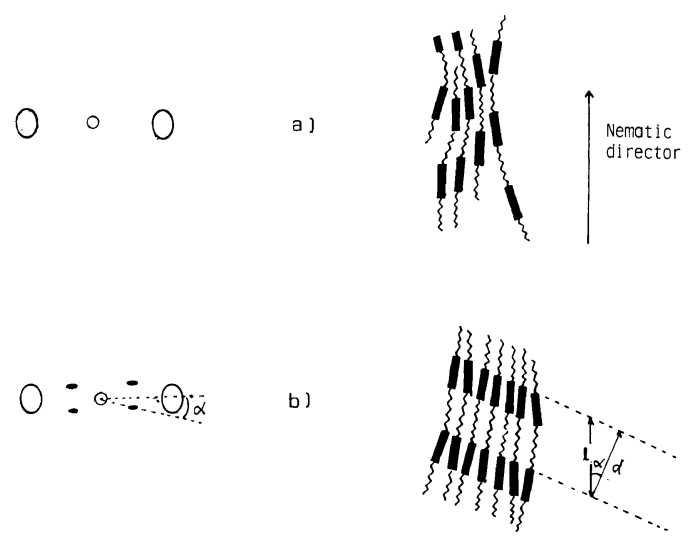

Figure 6. Features of a SAX pattern and of the proposed molecular organization for an oriented: a) "normal" nematic polymer corresponding to the odd members of poly $\left(4,4^{\prime}\right.$-dioxy-2,2'-dimethylazoxybenzenealkanedioyl); b) cybotactic micellar nematic polymer corresponding to the even members.

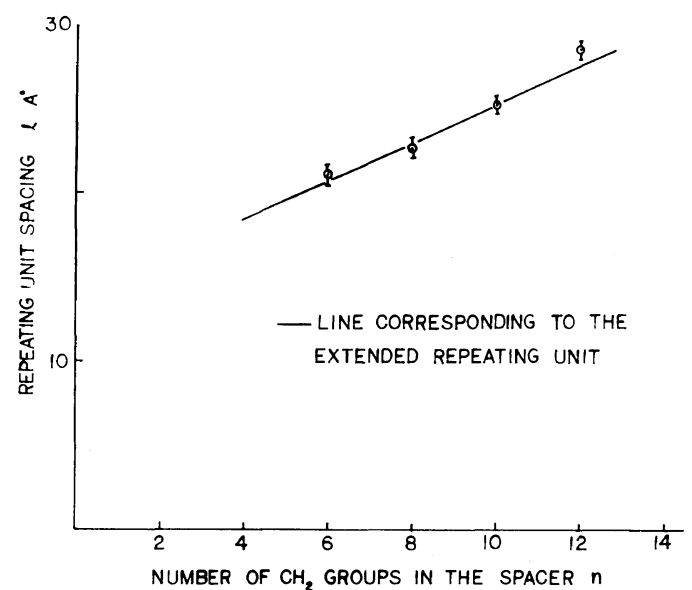

Figure 7. Length of the repeating unit from the SAX patterns of cybotactic nematic polyesters as a function $n$; polymers oriented and quenched in $\mathrm{H}=10 \mathrm{~T}$ at $T_{\text {red }}=$ 0.95 . Solid line corresponding to the length of the repeating unit in all-trans conformation. 


\section{A. Blumstein}

DeVries ${ }^{10}$ for LMLC while the absence of such pattern in the "odd" samples $(n=5,7,9$, and 11$)$ is revealing. The proposed model of cybotactic micellar clusters for even homologs ${ }^{11}$ and of an (odd) "normal" nematic ${ }^{12}$ is given in Figure 6. It is possible from the values of $\alpha$ and spacing $d$ to calculate, according to this model, the length of the repeating unit $l$ in the cybotactic micelle. The values of $l$ thus determined are within the error of the experiment $(0.5-0.8 \AA)$ identical to the length of the repeating unit extended in an all-trans conformation of the spacer. This is shown in Figure 7, where the solid line represents the spacings anticipated from an extended repeating unit and the points represent the experimental values of $d$ obtained from nematic mesophases oriented in a field of $10 T$ and quenched.

The X-ray data support, therefore, a rather extended conformation of the repeating unit for the even series in the nematic state. This has been confirmed by simulation of PMR spectra of DDA-9 by Martins et al. ${ }^{12}$ It was necessary to postulate a high order parameter $(S \sim 0.8)$ for both the mesogen and spacer in order to satisfactorily overlap the simulated and experimental spectra of DDA-9 (at $T_{\text {red }}$ of 0.96). This does not mean that the flexible spacer in the nematic phase of DDA-9 is in an all-trans conformation at temperatures of the mesophase. The presence of gauche conformers in the spacer is suggested by the lower values of isotropization enthalpy $\Delta H_{\mathrm{IN}}$ increments per methylene group than those expected from an all-trans conformation. ${ }^{9}$ One may note that a certain population of gauche conformers is compatible with the experimental uncertainty on the length of the repeating unit $( \pm 0.8 \AA)$ from SAX scattering. Recent $\mathrm{DMR}^{13}$ results on DDA-9 deuterium labeled in the spacer confirm the extended nature of the spacer while showing that it is not in an all-trans conformation. The spectrum is composed of two sets of quadrupolar splittings. The outer set corresponds to deuterons in $\alpha$ to the mesogen, and the inner corresponds to the eight remaining $\mathrm{CD}_{2}$ groups, all motionally equivalent. The outer splitting is proportional to the mesogen order parameters throughout the entire nematic range, suggesting a trans conformation with the $\mathrm{C}-\mathrm{D}$ bond on the average perpendicular to the main mesogen axis. The temperature dependence of the inner splitting relative to that of the mesogen order parameter indicates that the spacer disorders much faster than the mesogen as $T_{\mathrm{N} / \mathrm{I}}$ is approached. A similar observation can be made from PMR spectra by following the temperature dependence of the ratio of the spacer/mesogen dipolar splittings.

No data as to the extension of the flexible spacer in the odd series are as yet available. PMR spectra suggest that an extension of the spacer is also prevalent in this case, although the extent of it may be somewhat smaller than for the even series.

It is noteworthy to remember that both even and odd polyesters are randomly coiled in solution ${ }^{11}$ and in the isotropic melt. ${ }^{15}$ For solution in 1,1,2,2tetrachloroethane at $30^{\circ} \mathrm{C}$, the viscosity law for DDA-9 and AZA- 9 is respectively $[\eta]=4.65 \times 10^{-4}$ $M_{n}^{0.76}$ and $[\eta]=3.71 \times 10^{-4} M_{n}^{0.78}$, indicating a strongly solvated, randomly coiled chain for both polyesters. In the isotropic melt, the randomly coiled nature of polyesters of this type is revealed by the low value of the magnetic birefringence expressed by the Cotton-Mouton constant (CM) extracted from birefringence-field relationship $\Delta n=$ $(\mathrm{CM}) \lambda \mathrm{H}^{2}$. Typical values are between $(0.8$ 1.5) $10^{8} \mathrm{G}^{-2} \mathrm{~cm}^{-1} .{ }^{15}$ These values are similar to $\mathrm{CM}$ values for polystyrene in tetrachloroethylene or other flexible polymers and are $1-2$ orders of magnitude smaller than CM values of typical semirigid polymers such as DNA or polybenzamides. They are also close to values of $\mathrm{CM}$ for monomeric model compounds ${ }^{16}$ and clearly indicate that the persistence length of DDA-9 in the molten state does not substantially exceed the length of the monomeric unit. The passage from isotropic to the nematic state is for DDA-9 and AZA-9 a more first order phenomenon than for the LMLC analogs. This was already apparent from $\Delta H_{\mathrm{IN}}$ values, ${ }^{4}$ but is even more evident from measurements of $(\mathrm{CM})^{-1}$ dependence on $T$. From these measurements $T_{\mathrm{c}}-T^{*}$ intervals $\left(T^{*}\right.$, temperature at which the size of pretransitional nematic clusters $l \rightarrow \infty$; it corresponds to the virtual second order transition temperature) can be obtained.*1 The larger this interval, the more first order the isotropic $\rightarrow$ nematic

*1 In these measurements, the clearing temperature $T_{\mathrm{c}}$ is the temperature at which strong scattering of light due to the appearance of large nematic domains sets in. In polydisperse samples, $T_{\mathrm{c}}$ may not exactly coincide with $T_{\mathrm{IN}}$. 
Table III. Characteristics of the $\mathrm{I} \rightarrow \mathrm{N}$ transition for DDA-9 polymers and low molecular weight analogues ${ }^{\mathrm{a}}$

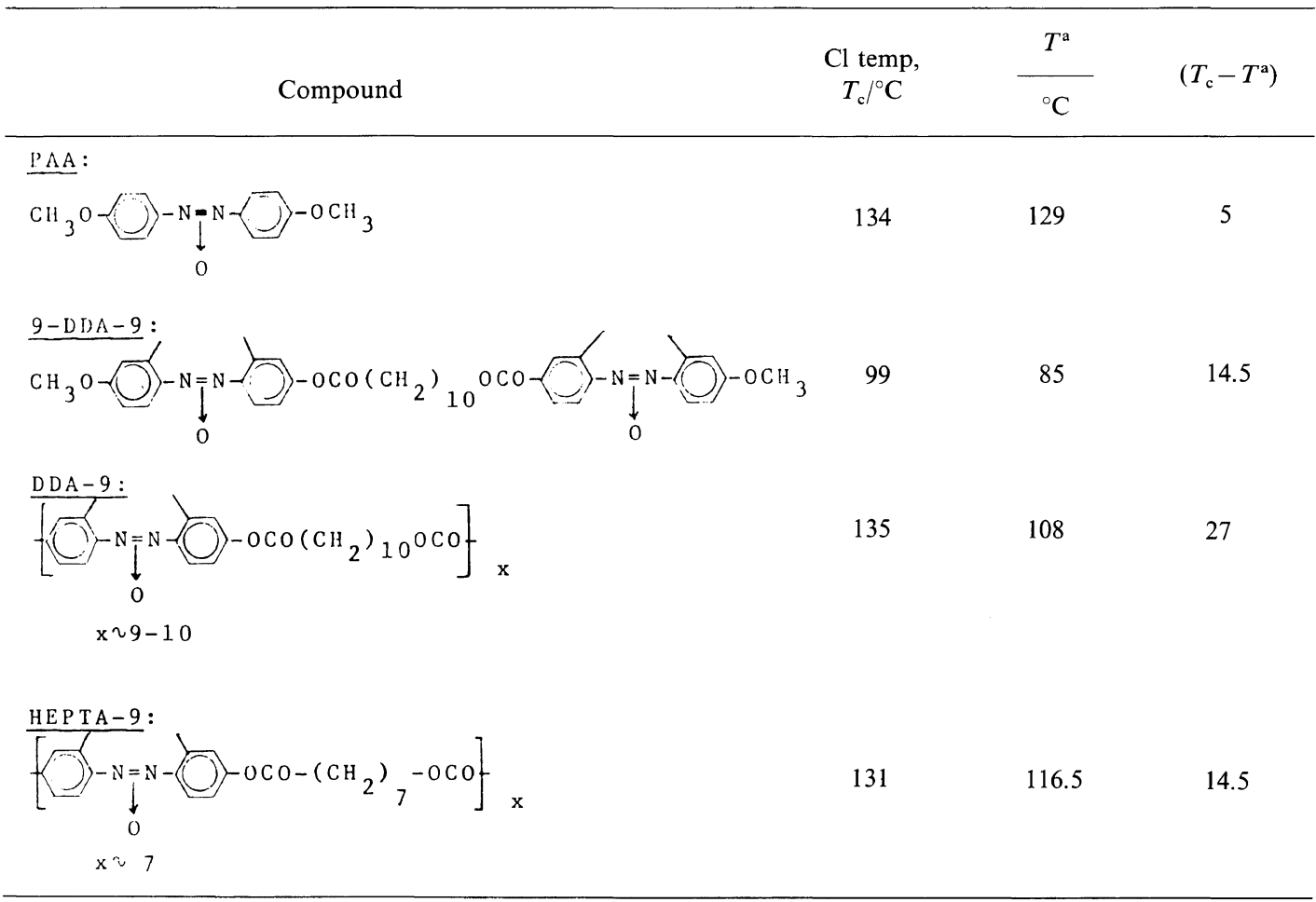

a Obtained in collaboration with Dr. G. Maret, Max Planck Inst., Hochfeld Magnet Laboratory, Grenoble, France. $^{16}$

transition. $\left(T_{\mathrm{c}}-T^{*}\right)$ also gives an indication about the extent of ordering in the isotropic melt in the vicinity of the clearing temperature (pretransitional ordering). Table III summarizes experimental data obtained by Maret ${ }^{16}$ on DDA-9 and AZA-9 PLC. One can see from Table III that $T_{\mathrm{c}}-T^{*}$ is $27^{\circ}$ for DDA- 9 vs. $14.5^{\circ}$ for AZA- 9 and only $5^{\circ}$ for the low molecular mass PAA.

These results indicate that pretransitional ordering is more extensive in cybotactic nematic PLC than in ordinary nematic PLC, which in turn display a stronger pretransitional ordering than a low molecular mass nematic such as PAA. It indicates that PLC, in contrast to LMLC, keep a certain degree of order beyond the actual N/I transition temperature. This is in agreement with the variation $S\left(T_{\text {red }}\right)$ obtained for DDA-9 and AZA-9.

Figure 8 gives the variation of the nematic order parameter measured by PMR for DDA-9 $(x=10)$ and AZA-9 $(x \sim 9-10)$ with temperature repre-

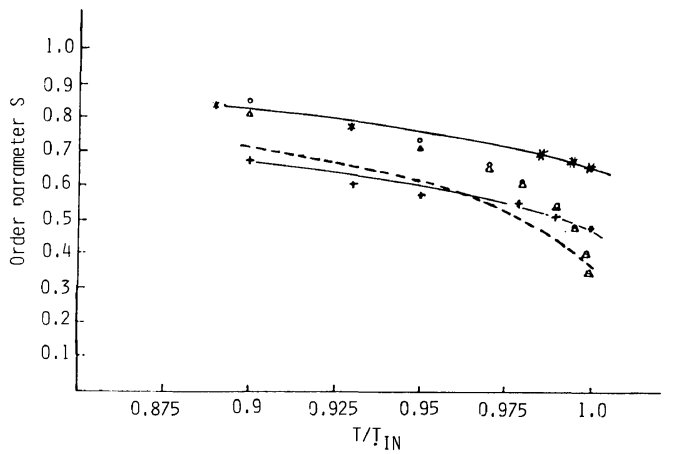

Figure 8. Order paramerer $S$ of the mesogen as determined from PMR as a function of $T_{\text {red. }}{ }^{*}$, DDA-9 $(n=10)$, fraction of $M_{n}=4000 ;+$, AZA-9 $(n=7)$, fraction of $M_{n}=4200 ; \bigcirc, 4,4^{\prime}$-bisbutoxyazoxybenzene ${ }^{17} ; \triangle$, $4,4^{\prime}$-bispentoxyazoxybenzene ${ }^{17}$; ---, theoretical curve for $\mathrm{PAA}^{18}$.

sented by continuous lines. Superimposed are experimental points representative of order parameter for low molecular mass analogs $4,4^{\prime}$-bisbutoxy- 
and 4,4'-bispentoxyazoxybenzenes, both of which give a cybotactic nematic mesophase. ${ }^{17}$ The interrupted line is $S\left(T_{\text {red }}\right)$ expected from Maier and Saupe treatment for PAA corrected for molecular dissymmetry. ${ }^{18}$ It is apparent immediately that in both polymers the $S(T)$ dependence is not overly strong as compared to LMLC, especially in the vicinity of the clearing point. The values for low molecular mass cybotactic nematics cross over from higher to lower order parameter dependence as $T$ increases, and drop dramatically at $T_{\text {red }}=1$. This suggests that cybotactic domains in low molecular mass nematics are unstable and dissociate progressively as the temperature increases. This dissociation appears complete at temperatures approaching the clearing point. In contrast, the cybotactic nematic structure in the DDA-9 is stable down to the immediate vicinity of $T_{\mathrm{IN}}$. The order parameter of the mesogen of ordinary nematic AZA-9 also appears to be stable, and the $S(T)$ dependence is very close to that given by the Meier and Saupe theory ${ }^{18}$ except in the vicinity of $T_{\mathrm{IN}}$. This suggests that in main chain PLC, as in systems with mesogenic side groups, the connectivity of mesogens furthers and stabilizes mesophase formation. This can be clearly seen in Figure 9 , where the order parameter $S_{\mathrm{c}}$ of DDA-9 at the clearing point obtained from measurements of magnetic birefringence through application of Landau-deGenne's theory, as given by Maret, ${ }^{16}$ is plotted as a function of the degree of polymerization $\left(\bar{X}_{n}\right)$. On the same graph, $S_{\mathrm{c}}$ obtained from PMR measurements is also plotted. One can see from Figure 9 that the values of $S_{\mathrm{c}}$ from PMR agree essentially with the values of $S_{\mathrm{c}}$ calculated from magnetic birefringence ${ }^{16}$ although a scattering is apparent at low $\bar{X}_{n}$. This scattering is not surprising in view of different $T_{\mathrm{c}}$ used in both methods due to the existence of a large biphase (see footnote*1). It is also clear from Figure 3 that $S_{\mathrm{c}}$ and $\Delta S_{\text {IN }}$ follow a similar dependence on the degree of polymerization $\bar{X}_{n}$ reflecting the order of the nematic phase at phase transition. Here, once more the cooperative nature of the formation of cybotactic micellar domains in DDA-9 is well apparent, stabilizing the cybotactic domain for $\bar{X}_{n}>10$.

SAX patterns of unoriented nematic mesophases of DDA-9 also indicate this effect in Figure 10. In the SAX pattern, a diffraction maximum at $16.5 \AA$ develops from a broad halo for $x>5$ to a well-

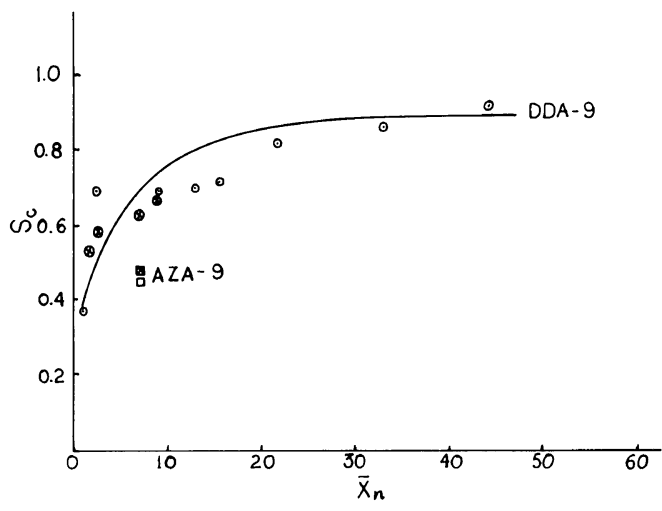

Figure 9. Order parameter $S_{\mathrm{c}}$ of the mesogen at the $\mathrm{N} / \mathrm{I}$ transition as a function of the number average degree of polymerization $\bar{X}_{n}$. DDA-9: $\otimes$, determined by PMR; $\odot$, computed from magnetic birefringence measurements ${ }^{16}$. AZA-9: $\square$, computed from magnetic birefringence ${ }^{16} ; \otimes$, determined by PMR.

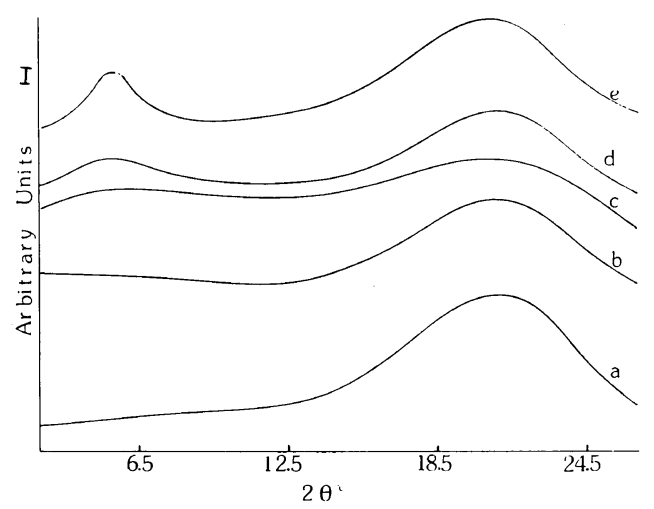

Figure 10. Evolution of the SAX pattern in unoriented DDA-9 quenched at $T_{\text {red }}=0.95$ as a function of number average degree of polymerization. AZA-9 $(n=7)$ : a), $\bar{X}_{n}=34$. DDA-9 $(n=10)$ : b), $\left.\bar{X}_{n}=4 ; \mathrm{c}\right), \bar{X}_{n}=5$; d), $\left.\bar{X}_{n}=10.4 ; \mathrm{e}\right), \bar{X}_{n}=40.6$.

defined peak at $x>10$. The transition seems to be progressive, and the sharpness and intensity of the peak increases with $\bar{X}_{n}$. No SAX pattern is recorded for AZA-9 regardless of $\bar{X}_{n}{ }^{7}$

These results corroborate the results of thermal analysis, the measurement of order parameter by PMR and magnetic birefringence. They all converge to indicate at $x>8-10$ the development for DDA-9 of a mesophase with a high chain alignment. The SAX patterns in the presence and also in the absence of a magnetic field suggest a layered organization of domains, the extent of which seems to 
increase with molecular mass. The question as to whether the structure of this mesophase is smectic or nematic can be legitimately asked. It was stated above that typical values of enthalpies and entropies of isotropization of DDA-9 are in the realm of smectic A phases of LMLC; so also is the high value of $S_{\mathrm{c}}$. The X-ray pattern of unoriented DDA-9, while indicating a layered periodicity, is not in tune with the somewhat sharper SAX spacing observed for smectics $\mathrm{A}$ and $\mathrm{C}$. The nematic nature of the cybotactic micellar mesophase is suggested by several facts. The viscosity of the system DDA-9 is rather low when compared to smectic mesophases and electro-hydrodynamic instabilities develop easily. DDA-9 and AZA-9 displayed for $M_{n}$ up to 10,000 Williams domains at low voltage, and for $M_{n}$ up to 4,000 dynamic scattering (DSM) typical of nematic mesophases. ${ }^{19,21}$ The miscibility of DDA-9 and PAA is total on heating and cooling. ${ }^{19}$ Also, DDA-9 and AZA-9 at $x>10$ have been found totally miscible. ${ }^{19}$ Hence, according to the miscibility criteria of Arnold and Sackmann, ${ }^{20}$ the mesophase of DDA-9 should be classified as nematic.

The miscibility of non-cybotactic PAA in cybotactic nematic DDA-9 can be also followed by NMR and DMR. The order parameter of mesogen of the polyester DDA-9 and of the PAA can be followed independently using mixtures of perdeuterated PAA-d14 and DDA-9. The order parameters of mesogen of DDA-9 in a mixture of $10 \%$ by weight of PAA-d14 dissolved in DDA-9 at various $T_{\text {red }}$, extracted from PMR spectra could thus be compared with order parameters of PAA-d 14 extracted from DMR spectra. The respective sets of points were very close to each other, although both sets of values were somewhat lower than the values of $S$ for the pure DDA-9. ${ }^{22}$ For mixtures containing 50\% PAA, the order parameter was found to decrease sharply and approach the value for PAA. Thus, it appears that cybotactic nematic domains in DDA-9 preserve their individuality even in the presence of small amounts of a foreign, non-cybotactic nematic component (PAA). This is also confirmed by X-ray analysis of copolymers containing up to $25 \%$ of spacer with widely different structure such as $25 / 75$ of dimethylglutaric acid/dodecanedioic acid (DMGA/DDA), ${ }^{7}$ which at this level of randomness still preserve a cybotactic organization. The X-ray and PMR data also suggest, nevertheless, that at concentrations of the foreign component beyond a critical value, cybotactic micelles are dissociated.

Acknowledgement. Thanks are expressed to $\mathbf{R}$. B. Blumstein, O. Thomas, M. Gautheir, F. Volino, G. Maret and J. Asrar for their participation in different parts of this work. This work was supported by the NSF Polymer Program under Grant DMR-8308939.

\section{REFERENCES}

1. A. Ciferri, "Polymer Liquid Crystals," A. Ciferri, W. R. Krigbaum and R. B. Meyer, Ed., Academic Press, New York, N. Y., 1982, p 63.

2. A. Blumstein, J. Asrar, and R. B. Blumstein, "Liquid Crystals and Ordered Fluids," Vol. 4, A. C. Griffin and J. F. Johnson, Ed., Plenum Press, New York, N. Y., 1984, p 311.

3. R. B. Lenz, in "Advances in Polymer Science," Springer Verlag, Berlin, in press.

4. R. B. Blumstein, E. M. Stickles, M. M. Gauthier, A. Blumstein, and F. Volino, Macromolecules, 17, 177 (1984).

5. F. Volino, J. M. Allonneau, A. M. Giroud-Godquin, R. B. Blumstein, E. M. Stickles, and A. Blumstein, Mol. Cryst. Liq. Cryst. (Lett.), 102, 21 (1984).

6. P. J. Flory and G. Ronca, Mol. Cryst. Liq. Cryst., 54, 289 (1979)

7. O. Thomas, Ph. D. dissertation, Department of Chemistry, University of Lowell, April 1984.

8. a. S. Vilasagar and A. Blumstein, Mol. Cryst. Liq. Cryst. (Lett.), 56, 263 (1980).

b. A. Blumstein and S. Vilasagar, Mol. Cryst. Liq. Cryst. (Lett.), 72, 1 (1981).

9. A. Blumstein and O. Thomas, Macromolecules, 15, 1264 (1982).

10. A. de Vries, Mol. Cryst. Liq. Cryst., 10, 219 (1970).

11. A. Blumstein, S. Vilasagar, S. Ponrathnam, S. B. Clough, G. Maret, and R. B. Blumstein, J. Polym. Sci. Polym. Phys. Ed., 20, 877 (1982).

12. a. A. Blumstein, R. B. Blumstein, M. M. Gauthier, O. Thomas, and J. Asrar, Mol. Cryst. Liq. Cryst. (Lett.), 92, 87 (1983).

b. A. Blumstein, O. Thomas, J. Asrar, P. Makris, S.

B. Clough, and R. B. Blumstein, J. Polym. Sci. Polym. Lett. Ed., 22, 13 (1984).

13. E. T. Samulski, M. M. Gauthier, R. B. Blumstein, and A. Blumstein, Macromolecules, 17, 479 (1984).

14. A. F. Martins, L. B. Ferreira, F. Volino, A. Blumstein, and R. B. Blumstein, Macromolecules, 16, 279 (1983)

15. A. Blumstein, G. Maret, and S. Vilasagar, Macromolecules, 14, 9523 (1981).

16. G. Maret, Polym. Prepr. Am. Chem. Soc., Div. 


\section{A. Blumstein}

Polym. Chem., 24, 2, 249 (1983).

17. E. C. Hansen and Y. R. Shen, Mol. Cryst. Liq. Cryst., 36, 193 (1976).

18. R. L. Humphries, P. G. James, and G. R. Lockhurst, J. Chem. Soc., Faraday Trans. II, 68, 1031 (1972).

19. A. Blumstein, H. W. Schmidt, O. Thomas, G. B Kharas, R. B. Blumstein, and H. Ringsdorf, Mol. Cryst. Liq. Cryst. (Lett.), 92, 271 (1984).

20. H. Arnold and H. Sackmann, Z. Phys. Chem., 213, 137 (1960).
21. J. M. Gilli, H. W. Schmidt, J. F. Pinton, P. Sixou, O. Thomas, G. Kharas, and A. Blumstein, Mol. Cryst. Liq. Cryst. (Lett.), 102, 49 (1984).

22. F. Volino and R. B. Blumstein, Mol. Cryst. Liq. Cryst., "Proceedings of InterEuropean Conference on Liquid Crystals," Bovec, Yugoslavia, 1984, in press.

23. R. B. Blumstein and E. M. Stickles, Mol. Cryst. Liq. Cryst. (Lett.), 82, 151 (1982). 